\title{
Crescimento e composição corporal em crianças e adolescentes com síndrome nefrótica córtico-dependente
}

\author{
Growth and body composition in children and adolescents with \\ steroid-dependent nephrotic syndrome
}

\begin{abstract}
Autores
Iza de Castro Oliveira ${ }^{1}$

Vera Maria Santoro

Belangero $^{2}$

'Departamento de Pediatria da Faculdade de Ciências Médicas da Universidade Estadual de Campinas - Unicamp - Campinas, SP, Brasil ${ }^{2}$ Departamento de Pediatria da Faculdade de Ciências Médicas da Universidade Estadual de Campinas - Unicamp - Campinas, SP, Brasil
\end{abstract}

Data de submissão: 21/05/2009 Data de aprovação: 28/09/2009

Correspondência para: Vera Maria Santoro Belangero

Rua Dr. Emílio Ribas 800

$1^{\circ}$ andar

CEP: 13025-141

Campinas, SP

As autoras declaram ter recebido apoio do FAEP - Fundo de Apoio ao

Ensino e Pesquisa - FCM Unicamp

\section{Resumo}

Objetivo: Avaliar crescimento e composição corporal em crianças e adolescentes com Síndrome Nefrótica Córtico-Dependente (SNCD). Material e Métodos: Foram incluídos todos os pacientes de 5 a 18 anos, em acompanhamento por pelo menos dois anos, com diagnóstico de SNCD. Foram coletados dados referentes a: tempo de tratamento, idade de início de tratamento, valores consecutivos do colesterol, albuminemia, proteinemia total, dose de uso de corticoide e peso, estatura e idade da primeira consulta. As avaliações antropométricas dobra cutânea triciptal e subescapular, índice de massa corpórea, circunferência muscular do braço, circunferência da cintura e z-escore de estatura/idade foram realizadas durante as consultas de rotina e realizadas somente quando se considerou a criança sem edema clinicamente visível. Estatística não paramétrica com $\mathrm{p}<0,05$. Resultados: Foram estudados 18 pacientes, 11 do sexo masculino $(61,1 \%)$, idade entre 6 e 16 anos $(12,22 \pm$ $2,98)$, tempo médio de tratamento de 6,75 $\pm 3,75$ anos. Os valores iniciais do z-escore foram significativamente maiores do que os finais $(-0,69 \pm 0,80$ e de $-2,07 \pm 1,61 ; \mathrm{p}=$ 0,003). A evolução individual do z-escore mostrou que houve diminuição em 14 (-1,37 $\pm 1,55$ ) e manutenção dos valores em quatro pacientes. Comparando-se vários parâmetros que poderiam ser responsáveis pela diferença de evolução, somente a proteinúria residual foi significativamente diferente. A medida da circunferência muscular do braço foi significativamente menor no grupo com perda de z-escore. Conclusões: Foi observado, na maioria dos pacientes, déficit de estatura e diminuição da massa magra, provavelmente associados à gravidade do quadro nefrótico, que necessitou de doses elevadas e prolongadas de corticoide.

Palavras-chave: síndrome nefrótica, crescimento, infância, adolescência, corticosteroides, composição corporal.

[J Bras Nefrol 2009;31(4):252-257]@Elsevier Editora Ltda.

\section{Abstract}

Objective: To assess growth and body composition in children and adolescents with steroid-dependent nephrotic syndrome (SDNS). Methods: All children aged 5 to 18 years diagnosed with SDNS and followed up at the Pediatric Nephrology Unit for at least two years were studied. The following data were collected from the medical records: duration of treatment; age at treatment onset; total dose of steroids (mg/ $\mathrm{kg}$ and $\mathrm{mg} / \mathrm{kg} / \mathrm{d}$ ); weight and height at the first visit; and the albuminemia, total proteinemia, and total cholesterol values. The anthropometric measures (triceps and subscapular skinfold thickness, body mass index, arm muscle circumference, waist circumference, and height/age z-score) were routinely determined when patients had no clinical edema. Nonparametric statistics was used with significance level of $p<0.05$. Results: The study comprised 18 patients (11 males), aged 6 to 16 years (mean age, $12.22 \pm 2.98)$, with a mean treatment duration of $6.75 \pm 3.75$ years. The initial height/ age $\mathrm{z}$-score values were significantly greater than the final ones $(-0.69 \pm 0.80$ and -2.07 \pm 1.61 , respectively; $p=0.003$ ). Considering the individual evolution, height/age $\mathrm{z}$-score decreased in 14 patients $(-1.37 \pm 1.55)$ and remained stable in four patients. Considering several parameters that could account for the difference in evolution, only residual proteinuria was significantly different. The arm muscle circumference was significantly smaller in the group with z-score decrease. Conclusion: Most patients had a deficit in height and a decrease in lean mass, probably associated with the severity of the nephrotic syndrome, which required high and prolonged corticosteroid doses.

Keywords: nephrotic syndrome, growth, childhood, adolescence, corticosteroids, body composition. 


\section{INTRODUÇÃO}

Embora a Síndrome Nefrótica (SN) na infância não tenha incidência elevada, ${ }^{1}$ sua prevalência não é desprezível pela frequência com que a doença recidiva em um mesmo paciente.

Dentre as inúmeras repercussões da doença no organismo, o efeito da $\mathrm{SN}$ sobre o crescimento é motivo de grandes preocupações e de muitas publicações na literatura médica. ${ }^{2-8}$ Sugestões de como diminuir os efeitos maléficos da terapêutica com corticoide e de como prevenir os distúrbios do metabolismo do cálcio e vitamina $\mathrm{D},{ }^{9}$ fatores primordiais associados ao déficit de crescimento na $\mathrm{SN}$, têm sido o objetivo de inúmeros estudos que visam à manutenção do crescimento na criança com $\mathrm{SN} .^{2-8}$

No entanto, a maioria dos estudos tem focalizado seus resultados em SN córtico-sensível, ${ }^{3,5,6}$ havendo dados muito escassos sobre a evolução de crianças e adolescentes com SN córtico-dependente e córticoresistente. ${ }^{4,7,8}$ Dessa forma, os resultados dos estudos não são uniformes quanto ao déficit de crescimento e aos seus fatores determinantes. Teoricamente, poderse-ia supor que estes dois últimos grupos de pacientes possam apresentar repercussões diretas e indiretas da SN mais graves do que os pacientes córtico-sensíveis, visto terem uma pior resposta à corticoterapia.

Da mesma forma, estudos sobre a composição corporal em SN têm sido realizados principalmente com casuísticas de crianças e adolescentes com SNCS. Nesses estudos, pode-se verificar que há uma diminuição da massa magra apendicular após o uso de altas doses de corticoide.

Este estudo tem como objetivo avaliar o crescimento e a composição corporal em crianças e adolescentes com Síndrome Nefrótica Córtico-Dependente (SNCD).

\section{Métodos}

Estudo retrospectivo e transversal. Foram avaliadas todas as crianças e adolescentes que faziam acompanhamento no Ambulatório de Nefrologia Pediátrica no Hospital das Clínicas da Universidade Estadual de Campinas - UNICAMP/FCM, com diagnóstico de Síndrome Nefrótica Córtico-Dependente (SNCD), no período de maio de 2006 a junho de 2007. A definição de córtico-dependência em SN seguiu o ISKDC (International Study of Kidney Disease in Children). ${ }^{9,10}$

Os critérios de exclusão foram: pacientes com SN córtico-sensível com recidivas frequentes e infrequentes e córtico-resistentes; aqueles com idade inferior a cinco anos ou acima de 18 anos; aqueles com menos de dois anos de acompanhamento no serviço; aqueles que não aceitaram participar do estudo; aqueles com hepatopatias e endocrinopatias; aqueles com SN secundária a doenças sistêmicas; e aqueles com taxa de filtração glomerular menor do que $60 \mathrm{~mL} / \mathrm{min} / 1,73 \mathrm{~m}^{2}$.

Todos os pacientes foram tratados com prednisona, de acordo com o protocolo aceito na literatura. ${ }^{11}$ Como terapêutica adicional foram utilizados esquemas individualizados que incluíram ciclofosfamida, ciclosporina A, levamisole ou micofenolato mofetil.

$\mathrm{O}$ projeto para esse estudo teve a aprovação do Comitê de Ética e Pesquisa da Faculdade de Ciências Médicas da Universidade Estadual de Campinas, em protocolo No 124/2006, em 17 de abril de 2006.

\section{DADOS OBTIDOS DO PRONTUÁRIO}

Foram coletados dos prontuários médicos os dados referentes a tempo de tratamento, idade de início de tratamento, valores consecutivos do colesterol, albuminemia, proteinemia total, dose de uso de corticoide e peso, estatura e idade da primeira consulta no ambulatório, para o cálculo do z-escore de estatura/ idade.

A dose de corticoide utilizada para cada paciente durante todo o período de acompanhamento foi obtida pela somatória de todas as doses desde o início do tratamento até a data atual de entrada no estudo (dose total). A seguir, determinou-se a média de peso do paciente no mesmo período, para que fosse feito o cálculo da dose em mg/kg. Após isso, esse valor foi dividido pelo número de dias equivalentes ao tempo de tratamento com corticoide, para a obtenção da dose em $\mathrm{mg} / \mathrm{kg} / \mathrm{dia} .^{5}$

\section{DADOS DETERMINADOS TRANSVERSALMENTE, NO MOMENTO ATUAL}

\section{DADOS ANTROPOMÉTRICOS}

As avaliações antropométricas foram realizadas durante as consultas de rotina e foram realizadas somente quando se considerou a criança sem edema clinicamente visível.

Para avaliar a condição nutricional atual, foram obtidos os dados de peso, estatura e dobra cutânea triciptal e subescapular. Crianças e adolescentes foram classificados de acordo com o percentil do IMC em eutrófico (IMC < p85), sobrepeso $(\mathrm{p} 85 \leq \mathrm{IMC}<$ p95) e obeso (IMC $\geq$ p95). ${ }^{11,12}$ Foi obtido o z-escore de estatura/idade para determinar o déficit de crescimento, sendo os pacientes classificados como tendo déficit de estatura aqueles cujo z-escore fosse $\leq-2,0 \mathrm{SD}$, com 
risco de déficit de estatura com z-escore -2,0 SD a $-1,0 \mathrm{SD}$, e sem déficit de estatura com z-escore >-1,0 SD. ${ }^{13}$ Utilizou-se para o cálculo da porcentagem de gordura a equação antropométrica da somatória das dobras cutâneas subescapular e triciptal segundo Slaughter et al. (1988). ${ }^{14}$

O estadiamento puberal foi avaliado por um único pediatra e classificado de acordo com a presença de caracteres sexuais secundários em ambos os gêneros ( $\mathrm{M}=$ mamas e $\mathrm{G}=$ testículos), como proposto por Marshall \& Tanner, ${ }^{15}$ para a realização do cálculo da porcentagem de gordura. ${ }^{14}$

A circunferência abdominal foi aferida com fita métrica inelástica em centímetros, no ponto médio entre a face externa da última costela e a ponta da crista ilíaca. Para obtenção desse ponto, o avaliado permaneceu em pé em movimento de expiração, com os braços estendidos ao longo do corpo. A classificação dessa medida será feita de acordo com o que foi determinado por McCarthy et al. (2001), ${ }^{16}$ para quem o valor considerado obesidade abdominal é acima do percentil 90.

A circunferência muscular do braço (CMB) foi utilizada para avaliar a reserva de tecido muscular e foi obtida a partir dos valores da circunferência do braço e da dobra cutânea triciptal. O cálculo de porcentagem de adequação de CMB foi realizado para classificar o estado nutricional dos pacientes. ${ }^{17}$

\section{AVALIAÇÃO URINÁRIA}

Coletou-se uma amostra da urina para a determinação da razão proteína/creatinina, que foi avaliada no Laboratório de Patologia Clínica do Hospital das Clínicas - Unicamp.

\section{ANÁLISE ESTATÍSTICA}

Foi utilizado o programa SPSS com os seguintes testes: a prova U de Mann-Whitney, para comparações entre grupos; Teste Wilcoxon, para comparações ao longo do tempo; e Teste Qui-Quadrado, para avaliação da distribuição de frequência.

$\mathrm{O}$ valor de $\mathrm{p}$ considerado foi menor ou igual a 0,05 .

\section{Resultados}

Foram estudados 18 crianças e adolescentes, 11 do sexo masculino $(61,1 \%)$, com idade entre 6 e 16 anos $(12,22 \pm 2,98$ anos), com tempo médio de tratamento de 6,75 $\pm 3,75$ anos. A distribuição dos pacientes de acordo com os valores iniciais e finais do z-escore estatura/idade está demonstrada na Figura 1.
Figura 1. Distribuição da casuística por diagnóstico de déficit de estatura, segundo z-escore de estatura/ idade $(E / I)$.

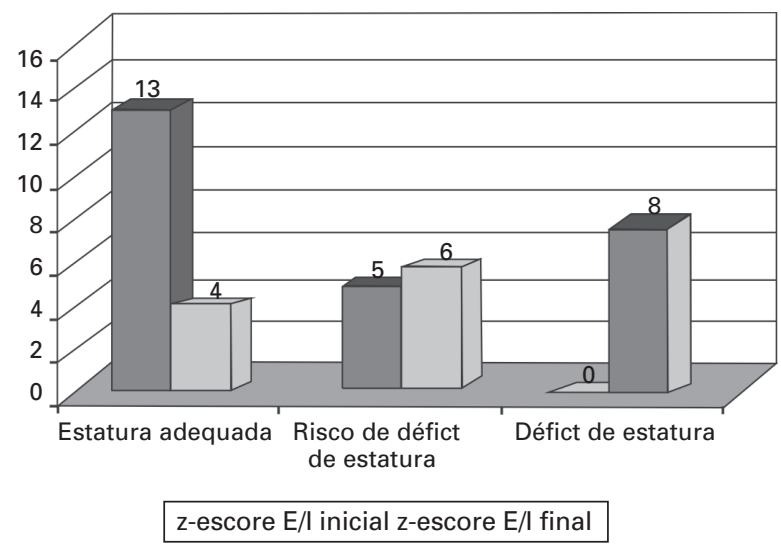

Os valores do z-escore inicial foram significativamente maiores que os finais, sendo respectivamente de $-0,69 \pm 0,80$ e de $-2,07 \pm 1,61(p=0,003)$. Houve déficit de estatura em oito pacientes, considerando-se o valor mais recente do z-escore de estatura/idade. A evolução individual do valor do z-escore é mostrada na Figura 2, na qual se observa que 14 pacientes tiveram diminuição dos valores, sendo o valor médio de perda de $-1,37 \pm 1,55$.

Figura 2. Valores iniciais e finais do z-escore de estatura/idade de pacientes com síndrome nefrótica córtico-dependente e córtico-resistente.

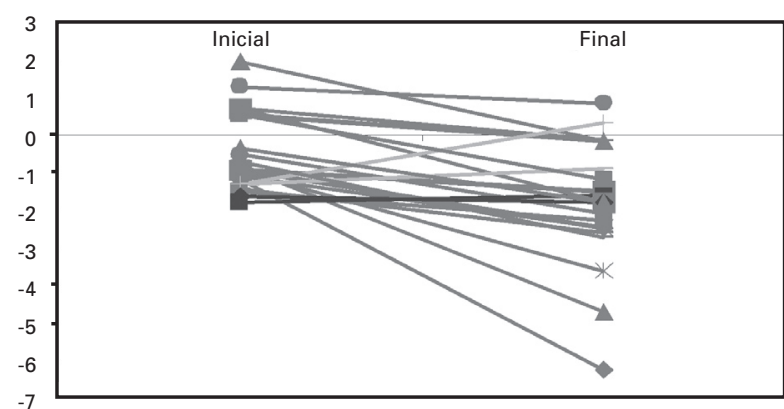

Dentro do grupo dos 14 pacientes com piora do z-escore de estatura/idade, havia sete de cada gênero, sendo que quatro tinham atingido a estatura final.

Comparando-se vários parâmetros que poderiam ser responsáveis pela diferença de evolução do z-escore, pode-se observar que o grupo com diminuição dos valores apresentou maior dose de uso de corticoide, 
idade mais precoce de início da $\mathrm{SN}$, menor valor médio da albuminemia e da proteinemia total e valores maiores da colesterolemia e do tempo de tratamento, mas com diferenças estatisticamente não significativas (Tabela 1). No entanto, a proteinúria residual foi significativamente menor nos pacientes com manutenção do z-escore (Tabela 1).

Comparando-se os parâmetros antropométricos nos dois grupos, pode-se observar que há diminuição estatisticamente significativa nos valores de adequação da circunferência muscular do braço no grupo com diminuição do z-escore de estatura/idade. Outros parâmetros antropométricos não tiveram diferença significativa (Tabela 2).

\section{Discussão}

Nessa casuística de crianças e adolescentes com SNCD com tempo longo de tratamento, foi observado que houve déficit importante de crescimento na maioria dos pacientes.

Há poucos relatos na literatura a respeito do crescimento em SNCD. Rees et al. (1988), ${ }^{6}$ em casuística semelhante, concluíram que houve piora significativa do escore de desvio-padrão para estatura tanto com relação ao tempo de tratamento quanto em função da idade do paciente, sendo mais intensa no sexo masculino. A perda de desvio-padrão foi de $-0,47$, com variação de $-0,84$ a $-0,11$. Os valores absolutos de

\begin{tabular}{|c|c|c|c|c|}
\hline \multirow[t]{2}{*}{ Tabela 1} & \multicolumn{4}{|c|}{$\begin{array}{l}\text { COMPARAÇÃO DE DOSE DE CORTICOIDE, IDADE DE INÍCIO DA SN, MÉDIA DE ALBUMINENIA, MÉDIA DA COLESTEROLEMIA, } \\
\text { MÉDIA DA PROTEINEMIA TOTAL, TEMPO DE TRATAMENTO E PROTEINÚRIA ATUAL ENTRE OS GRUPOS COM DIMINUIÇĀO } \\
\text { DO Z-ESCORE DE ESTATURA/IDADE E O GRUPO COM MANUTENÇÃO DO Z-ESCORE DE ESTATURA/IDADE }\end{array}$} \\
\hline & & $\begin{array}{l}\text { Diminuição do z-escore } \\
\qquad(\mathrm{n}=14)\end{array}$ & $\begin{array}{l}\text { Manutenção do z-escore } \\
\qquad(\mathrm{n}=4)\end{array}$ & $\mathrm{p}$ \\
\hline \multicolumn{2}{|c|}{ Dose de corticoide (mg/kg/dia) } & $0,57 \pm 0,28$ & $0,51 \pm 0,25$ & 0,79 \\
\hline \multicolumn{2}{|c|}{ Dose total de corticoide (mg/kg) } & $1567,20 \pm 298,57$ & $936,52 \pm 393,74$ & 0,32 \\
\hline \multicolumn{2}{|c|}{ Idade início da SN } & $5,5 \pm 4,0$ & $7,2 \pm 4,5$ & 0,44 \\
\hline \multicolumn{2}{|c|}{ Média de albuminemia } & $2,52 \pm 0,85$ & $3,12 \pm 0,43$ & 0,19 \\
\hline \multicolumn{2}{|c|}{ Média da colesterolemia } & $352,73 \pm 147,27$ & $240,04 \pm 42,61$ & 0,07 \\
\hline \multicolumn{2}{|c|}{ Média da proteinemia total } & $5,12 \pm 0,97$ & $6,00 \pm 0,98$ & 0,13 \\
\hline \multicolumn{2}{|c|}{ Tempo de tratamento (anos) } & $8,25 \pm 4,75$ & $6,03 \pm 3,45$ & 0,38 \\
\hline \multicolumn{2}{|c|}{ Proteinúria atual (Razão Prot/Crea) } & $6,56 \pm 6,05$ & $0,33 \pm 0,27$ & 0,01 \\
\hline
\end{tabular}

Razão Prot/Crea = Razão proteína/creatinina

\begin{tabular}{|c|c|c|c|c|}
\hline \multirow[t]{2}{*}{ Tabela 2} & \multicolumn{4}{|c|}{$\begin{array}{l}\text { COMPARAÇÃO DOS DADOS ANTROPOMÉTRICOS: ADEQUAÇÃO DA CIRCUNFERÊNCIA MUSCULAR DO BRAÇO, ADEQUAÇÃO } \\
\text { DE TRÍCEPS, ÍNDICE DE MASSA CORPÓREA, PORCENTAGEM DE GORDURA E CIRCUNFERÊNCIA ABDOMINAL, NOS GRUPOS } \\
\text { COM DIMINUIÇÃO DO Z-ESCORE DE ESTATURA/IDADE COM O GRUPO DE MANUTENÇÃO DO Z-ESCORE DE ESTATURA/IDADE }\end{array}$} \\
\hline & & $\begin{array}{l}\text { Diminuição do z-escore } \\
\qquad(\mathrm{n}=14)\end{array}$ & $\begin{array}{l}\text { Manutenção do z-escore } \\
\qquad(\mathrm{n}=4)\end{array}$ & $\mathrm{p}$ \\
\hline \multicolumn{2}{|c|}{ Adequação de CMB } & $87,55 \pm 15,47$ & $106,18 \pm 7,57$ & 0,005 \\
\hline \multicolumn{2}{|c|}{ Adequação de tríceps } & $130,37 \pm 77,17$ & $120,09 \pm 48,96$ & 0,959 \\
\hline \multicolumn{2}{|l|}{ IMC } & $20,14 \pm 4,33$ & $23,99 \pm 7,02$ & 0,327 \\
\hline \multicolumn{2}{|c|}{ Porcentagem de gordura } & $20,57 \pm 10,88$ & $23,92 \pm 14,56$ & 0,721 \\
\hline \multicolumn{2}{|c|}{ Circunferência abdominal } & $71,24 \pm 11,29$ & $76,62 \pm 15,91$ & 0,645 \\
\hline \multicolumn{2}{|c|}{ Obesidade (S/N) } & $3 / 11$ & $0 / 4$ & 0,446 \\
\hline
\end{tabular}

CMB - Circunferência Muscular do Braço; IMC - Índice de Massa Corpórea 
z-escore para cada paciente não foram apresentados no estudo, mas, a partir das figuras que constam no artigo, pode-se observar que, no sexo masculino, o valor médio do z-escore de estatura/idade para maiores de 12 anos foi de 0,70 negativo. Foi também demonstrado neste estudo que havia bloqueio na liberação do hormônio de crescimento $(\mathrm{GH})$ e retardo no início da puberdade. As doses cumulativas de corticoide não foram determinadas no estudo, nem os valores da albuminemia ou da proteinúria residual, para que fosse possível a comparação desses resultados, mais favoráveis com relação à perda de z-escore de estatura/ idade, com os do presente estudo.

Por outro lado, no estudo de Donatti et al. (2003), ${ }^{3}$ também pode ser distinguido um grupo com diagnóstico final de SNCD ( $\mathrm{n}=13)$. Nesse grupo, houve seis pacientes que evoluíram com ganho e sete pacientes que tiveram perda do escore de estatura. Não foi possível identificar as doses cumulativas individuais de corticoterapia, mas no grupo de pacientes com melhora da estatura, a dose média cumulativa de corticoide foi de $980 \mathrm{mg} / \mathrm{kg}$, e para o grupo com retardo de crescimento, foi de $1.384 \mathrm{mg} / \mathrm{kg}$, sendo que três dos sete usaram média de $2.100 \mathrm{mg} / \mathrm{kg}$. No presente estudo, a dose cumulativa dos pacientes com manutenção da estatura é muito semelhante à do subgrupo correspondente do estudo anteriormente citado (936,5 $\mathrm{mg} / \mathrm{kg}$ ) e a dose média dos paciente com déficit de estatura é intermediária $(1.567,2 \mathrm{mg} / \mathrm{kg})$. Assim, os dados do presente estudo estão de acordo com os de Donatti et al. $(2003)^{3}$ e reafirmam a importância da dose cumulativa do corticoide na gênese do retardo de crescimento em pacientes com SNCD.

É necessária também a comparação dos resultados do presente estudo com os de Emma et al., em 2003, no qual foram estudadas 56 crianças com SNCD ou recidivante frequente, com tempo mínimo de seis anos de acompanhamento. O regime de tratamento seguiu o esquema clássico de corticoterapia em $\mathrm{SN}$, e a perda do escore de estatura foi de 0,49 $\pm 0,6$ até a fase puberal, e de $0,92 \pm 0,80$ na estatura final dos pacientes. A dose cumulativa de corticoide foi de $1.560 \pm$ $720 \mathrm{mg} / \mathrm{kg}$ no sexo masculino e de $1.310 \pm 750 \mathrm{mg} /$ $\mathrm{kg}$ no feminino, sendo o único fator de risco para o déficit de crescimento. Comparando-se estes dados com os do presente estudo, observa-se que embora as doses totais de corticoide sejam muito semelhantes, o déficit de estatura foi maior no presente estudo.

Como justificativa, pode-se sugerir que os pacientes de Emma et al. tinham melhor resposta à corticoterapia, visto que o número médio de recidivas/ano foi de 1,8 , valor que mais indica um caso de recidiva infrequente do que de córtico-dependência. Além disso, mais da metade dos casos (29/56 pacientes) apresentaram remissão permanente. Assim, é possível que esses pacientes tenham tido mais tempo fora de corticoterapia e maior oportunidade de recuperação do déficit de crescimento.

Por outro lado, se compararmos os resultados do estudo de Scharer et al. (1999) ${ }^{4}$ com crianças e adolescentes com SNCR, pode-se observar que os valores de proteinemia total e albuminemia do presente estudo $(5,31 \mathrm{~g} \%$ e $2,66 \mathrm{~g} \%$, respectivamente) são semelhantes aos dos pacientes do estudo citado $(5,35 \mathrm{~g} \%$ e $2,68 \mathrm{~g} \%$, respectivamente). No entanto, o déficit de estatura ao final de longo tempo de acompanhamento do citado estudo é muito menor do que o aqui apresentado. ${ }^{4}$ No estudo citado, os pacientes fizeram uso de corticoide na dose total média de $232 \mathrm{mg} / \mathrm{kg}$ e, na avaliação de um subgrupo de 16 pacientes que não estavam em uso da corticoterapia, foi possível observar uma correlação significativa entre a perda do escore de estatura com valores diminuídos de proteinemia total e albuminemia. Neste estudo, não foi observada uma correlação significativa entre os parâmetros proteicos e o retardo de crescimento, embora o grupo com manutenção do crescimento apresente valores médios de albuminemia e proteinemia total maiores do que o grupo com déficit de estatura. Provavelmente, o tamanho da casuística poderia justificar em parte essa diferença dos resultados, mas, mais provavelmente, esse encontro reflete o efeito negativo preponderante sobre o crescimento da corticoterapia prolongada e em dose elevada. Dessa forma, "o custo" da melhora da albuminemia e proteinemia pelo uso da corticoterapia deve ser fortemente questionado.

Além disso, foi observado que os pacientes com manutenção do crescimento apresentaram níveis significativamente menores de proteinúria, o que, certamente, está associado à menor dose de corticoide, aos valores melhores de colesterol e, provavelmente, representam casos de SNCD de melhor evolução do que o grupo com déficit de estatura. Essa melhor evolução teve reflexo também importante na avaliação do estado nutricional, expressado pela adequação da circunferência do braço no grupo dos pacientes com manutenção do crescimento, que foi significativamente diminuída no outro grupo. Esses dados estão de acordo com os apresentados por Foster et al. (2004), ${ }^{5}$ que demonstraram diminuição significativa na massa magra apendicular em crianças tratadas com dose elevada de corticoide em SN. 
Dentre os fatores descritos na literatura que relacionam o déficit de crescimento com a $\mathrm{SN}$, o uso da corticoterapia, a hipoalbuminemia e hipoproteinemia total, os distúrbios no metabolismo de cálcio e vitamina $\mathrm{D}$ e a diminuição da taxa de filtração glomerular são considerados os mais importantes. Na SNCD, como não é frequente a diminuição do ritmo de filtração glomerular e os efeitos sobre o metabolismo de cálcio e vitamina $\mathrm{D}$ podem ser suprimidos com uso regular de vitamina D e cálcio, o uso da corticoterapia passa a ser o fator exclusivo do déficit de crescimento nesse grupo de pacientes, que tende a ocorrer de forma mais grave que nos pacientes com SNCR. Assim, o benefício da corticoterapia na SNCD para a obtenção da melhora da proteinúria e da hipoalbuminemia deve ser questionado em função dos seus efeitos colaterais irreversíveis sobre o crescimento e a composição corporal.

\section{REFERÊNCIAS}

1. Andrade OVB, Mello VR, Martini D. Glomerulopatias. In: Toporovski J, Mello VR, Martini D, Benini V, Andrade OVB. Editor. Nefrologia Pediátrica. $2^{\circ}$ edição. Rio de Janeiro: Guanabara Koogan, 2006, pp. 145-207.

2. Huang YT, Yang LY. Follow-up of linear growth of body height in children with nephrotic syndrome. J Microbial Immunol Infect 2006; 39:422-25.

3. Donatti TL, Koch VH, Fujimura MD, Okay Y. Growth in steroid-responsive nephrotic syndrome: a study of 85 pediatric patients. Pediat Nephrol 2003; 18:789-95.

4. Schärer K, Essigmann HC, Schaefer F. Body growth of children with steroid-resistant nephrotic syndrome. 1999; 13:828-34.

5. Foster BJ, Shults J, Zemel BS, Leonard MB. Interactions between growth and body composition in children treated with high-dose chronic glucocorticoids. Am J Clin Nutr 2004; 80:1334-41.
6. Rees L, Greene SA, Adlard P et al. Growth and endocrine function in steroid sensitive nephrotic syndrome. Arch Dis Childh 1988; 63:484-90.

7. Lan CN, Arneil GC. Long-term dwarfing effects of corticosteroid treatment for childhood nephrosis. Arch Dis Childh 1968; 43:589-94.

8. Emma F, Sesto A, Rizzoni G. Long-term linear growth of children with severe steroid-responsive nephrotic syndrome. Pediatr Nephrol 2003; 18:783-8.

9. Donatti TL, Koch VH, Fujimura MD, Okay Y. Revisão: crescimento da criança e do adolescente com síndrome nefrótica idiopática. J Bras Nefrol 2001; 23:32-9.

10. International Study of Kidney Disease in Children. Nephrotic syndrome in children: Prediction of histopathology from clinical and laboratory characteristics at time of diagnosis. Kidney Int 1978; 13:159-65.

11. Broyer M, Meyrier A, Niaudet P, Habib R. Minimal changes and focalsegmental glomerular sclerosis. In: Oxford Textbook of Clinical Nephrology. 2nd Edition. London: Oxford Medical Publications, 1998, pp. 493537.

12. Kuczmarski RJ, Ogden CL, Guo SS et al. 2000 CDC growth charts for the United States: methods and development. Vital health stat 2002; 11:1-190.

13. World health organization. WHO Child growth standards: length/height for age, weight for age, weight for length, weight for height and body mass index for age. Methods and development: WHO (nonserial publication). Geneva, Switzerland: WHO, 2006.

14. Slaughter MH, Lohman TG, Boileau RA et al. Skinfold equations for estimation of body fatness in children and youth. Human Biology 1988; 60:709-23.

15. Marshall WA, Tanner JM. Puberty. In: Bavid JA, Dobbing J. Scientific foundations of paediatric. Philadelphia: W.B. Saunders, 1974, pp. 124-51.

16. McCarthy HD, Jarrett KV, Crawley HF. The development of waist circumference percentiles in British children aged 5.0 - 16.9 y. Eur J of Clin Nutr 2001; 55:902-7.

17. Frisancho AR. Anthropometric standards for the assessment of growth and nutritional status. Ann Arbor, Michigan: University of Michigan Press, 1990. 